\title{
Good clinical practice in dubious head trauma - the problem of retained intracranial foreign bodies
}

\author{
Bernhard R Fischer' \\ Yousef Yasin² \\ Markus Holling ${ }^{2}$ \\ Volker Hesselmann ${ }^{3}$ \\ 'Department of Neurotraumatology, \\ BG-University Hospital \\ Bergmannsheil, Ruhr-University \\ Bochum, Bochum, Germany; \\ ${ }^{2}$ Department of Neurosurgery, \\ ${ }^{3}$ Institute for Clinical Radiology, \\ University Hospital of Muenster, \\ Muenster, Germany
}

This article was published in the following Dove Press journal:

International Journal of General Medicine

18 October 2012

Number of times this article has been viewed
Objective: In young people, traumatic head and brain injuries are the leading cause of morbidity and mortality. In some cases, no neurological deficits are present, even after penetrating trauma. These patients have a greater risk of suffering from secondary injuries due to secondary infections, brain edema, and hematomas. We present a case report which illustrates that brain injuries that do not induce neurological deficits can still result in a fatal clinical course and death, with medicolegal consequences.

Clinical presentation: A 19-year-old patient was admitted to hospital suffering from a head injury due to an assault. He reported that he was attacked from behind. Medical examination showed no neurological deficits, and only a small occipital wound. Neuroimaging of the cranium revealed that a knife blade was penetrating the cranial bone and touching the superior sagittal sinus.

Intervention: After removing the foreign body, magnetic resonance imaging showed that the superior sagittal sinus remained open.

Conclusion: We want to stress that possible problems can arise due to the retention of objects in the cranium, while also highlighting the risk of superficial clinical examination.

Keywords: head trauma, guidelines, retained object, neuroimaging

\section{Introduction}

Traumatic head and brain injuries, including penetrating head injuries, are the leading cause of morbidity and mortality in young people. ${ }^{1,2}$ However, in some patients these head injuries do not affect neurological function, even if the foreign body is retained in the brain parenchyma. ${ }^{2-7}$ When no neurological deficits are presented and no foreign body is visible during inspection, these cases are sometimes wrongly classified as a mild head injury. Furthermore, existing guidelines in the treatment of mild cranial traumas do not include protocols for neuroimaging examination. ${ }^{8-10}$ Secondary infection, brain edema, and hematomas can result in a fatal clinical course and death with medicolegal consequences. ${ }^{3,4,11,12}$ In 1997, a series of patients presented with transcranial stab wounds; it was discovered that those with retained knife blades were shown to be at high risk for secondary morbidity and mortality. ${ }^{7}$ Guidelines do not currently exist for this group of patients, or for the necessary neuroimaging examinations or surgical approaches required to treat these individuals. We therefore present a special case of a penetrating head injury that was initially overlooked, and discuss the diagnostic and therapeutic procedures that were used within the context of the existing guidelines. ${ }^{9}$
Correspondence: Bernhard R Fischer Department of Neurotraumatology, BG-University Hospital Bergmannsheil, Ruhr-University Bochum, Bürkle-de-laCamp-Platz I, 44789 Bochum, Germany Tel +492343023870

Fax +49234302913870

Email bernhard.fischer@bergmannsheil.de 


\section{Clinical presentation}

A 19-year-old patient suffered from a head injury due to an assault that occurred from behind. He was admitted to an emergency department to have a dressing applied to a small occipital laceration. Clinical examination revealed no loss of consciousness, nausea, vomiting, or any other neurological deficit; however, a hard and bloody eschar was noted on his scalp in the occipital region (Figure 1). Inspection of the wound conducted prior to the intended suturing demonstrated a foreign metallic body in the wound that could not be removed. The patient was admitted into our emergency unit, and cranial computerized tomography (CT) was performed, revealing a knife blade penetrating the cranial bone. Additionally, CT angiography demonstrated the foreign body touching, or potentially penetrating, the bridging veins and the superior sagittal sinus (Figure 2), which appeared to be patent. At that time, and in view of the neuroimaging results, it was unclear as to whether the knife had harmed the sinus, and if removal of the knife blade would provoke massive bleeding. In view of the possibly lacerated dorsal part of the sinus, we decided to perform a surgical intervention.

\section{Neurosurgical intervention}

Emergency surgical treatment was performed. Removing the knife blade from the bone would be associated with the overwhelming risk of reinjuring the superior sagittal sinus.

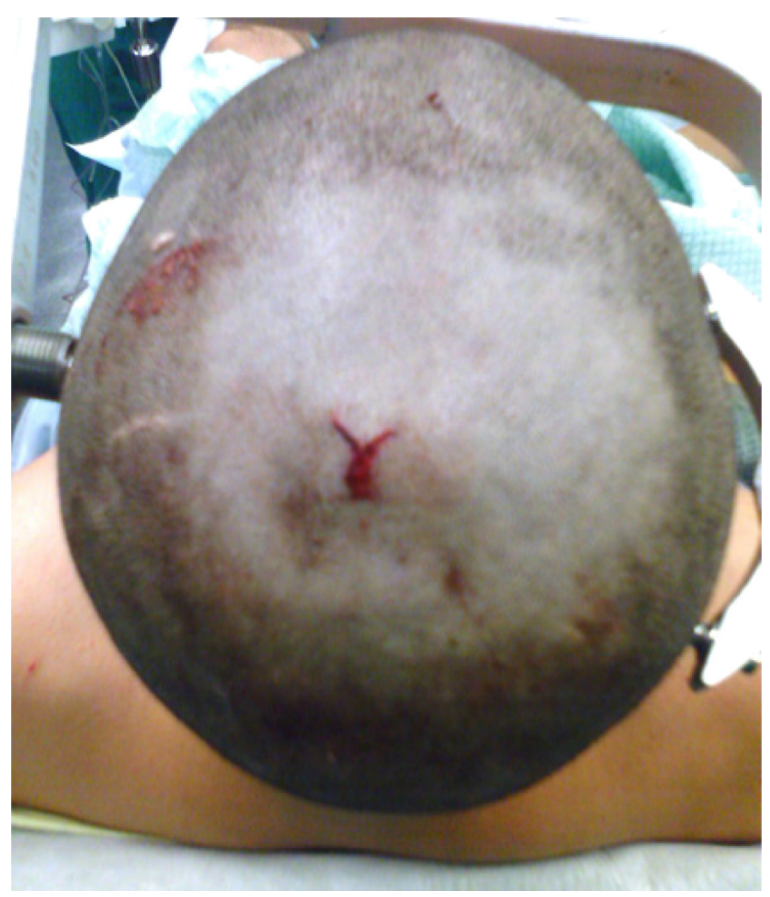

Figure I Inspection of the head showing only a small open wound with a little swelling. Note: The foreign body is not visible.

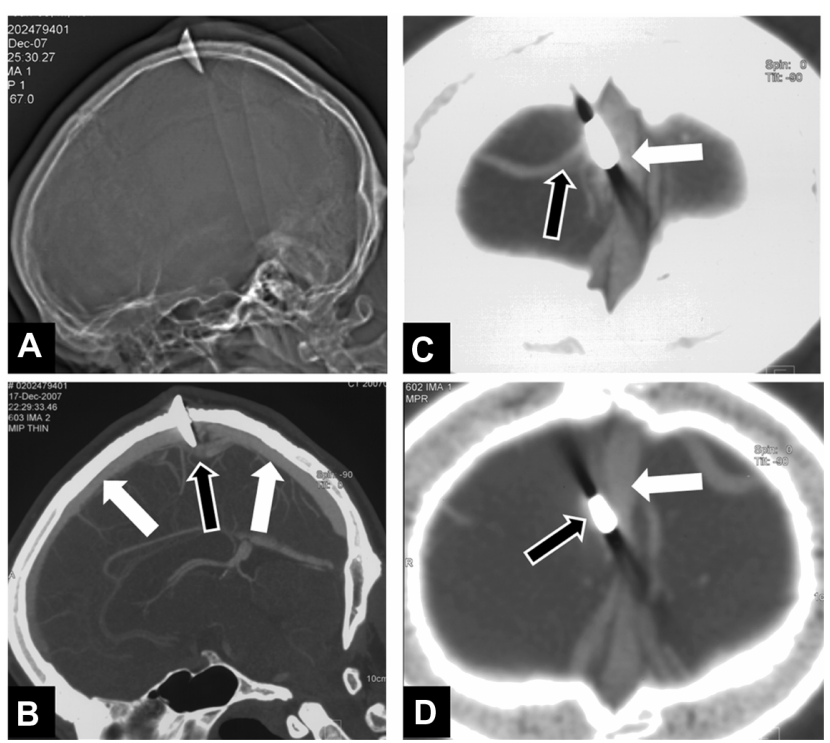

Figure $2 \mathrm{CT}$ and $\mathrm{CT}$ angiography of the brain. CT of the brain revealing a metallic foreign body penetrating the skull bone (A). In CT angiography (B-D), the superior sagittal sinus (white arrows) as well as the bridging veins (black arrows) seem to be contacted, or even injured by the knife blade.

Abbreviation: CT, computerized tomography.

Consequently, we performed a craniotomy that extended beyond the superior sagittal sinus while the knife blade remained in situ (Figure 3). After vertical elevation of the bony fragment, only marginal bleeding from the sinus occurred. The sinus leakage was covered with autologous material. To remove the knife blade from the bone, parts of the bone had to be removed. The remaining bone fragment was replaced and fixed with titanium clamps (Figure 4). Due to suspected inflammation, antibiotics were prescribed for 5 days following the procedure.

\section{Clinical course}

The postoperative clinical course was uneventful. No neurological deficits occurred, and there were no clinical or

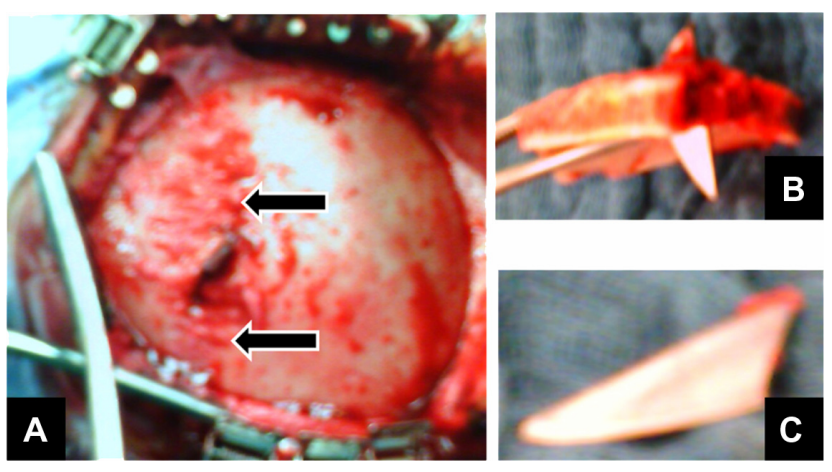

Figure 3 Intraoperative view. Intraoperative view, demonstrating the knife blade protruding only a few millimeters above the skull surface (A and $\mathbf{B})$, penetrating the skull directly adjacent to the sagittal suture (black arrows), within the elevated bone fragment (B), and after removal (C). 


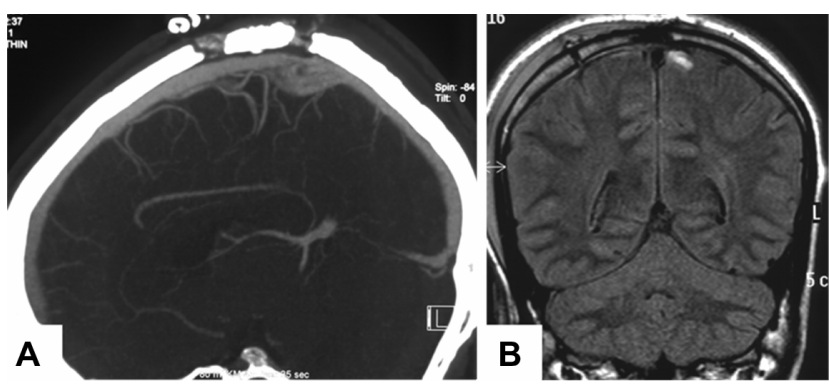

Figure 4 Postoperative cranial CT angiography and MRI. (A) Postoperative cranial CT angiography revealing a still-open superior sagittal sinus, as well as open bridging veins. (B) MRI of the brain showing that only a slight left parasagittal cortical hemorrhage is present after the trauma and treatment.

Abbreviations: $\mathrm{CT}$, computerized tomography; MRI, magnetic resonance imaging.

laboratory signs of inflammation. Postoperative neuroimaging revealed only minor intraparenchymal bleeding, but no other morphological changes (Figure 4). Importantly, the superior sagittal sinus and the bridging veins remained open. The patient was discharged from our hospital 7 days after injury.

\section{Discussion}

Traumatic head and brain injuries are significant causes for morbidity and mortality, especially in young people. Classification of these traumas depends on their underlying mechanisms, their clinical presentation, and pathological neuroanatomical changes. ${ }^{10}$ In order to provide better treatment and to reduce possible secondary damages, many guidelines have been developed, which are mostly related to severe head injuries. ${ }^{9}$ For patients with head injuries, but presenting with or without mild neurological deficits, standard practices remain unclear. ${ }^{13}$

Existing guidelines do not currently recommend neuroimaging for diagnostic purposes among this group of patients, and at best they suggest monitoring patients overnight for observation. ${ }^{9}$ Although it is advised that a CT scan of the cerebrum be performed in patients with suspected open or depressed skull fracture, ${ }^{9}$ our patient would not have received any radiological diagnostics due to his inconspicuous clinical status and his negligible wound. However, this approach could have medicolegal consequences, as reported previously. $^{4}$

Thus, good practice suggests that every patient with an open wound be inspected carefully - a claim that should be self-evident. If there is any risk of existing penetrating or depressed skull trauma, CT imaging should be performed, even in patients with a Glasgow Coma Scale sum score of 14 or $15 .{ }^{14}$ Previous studies have demonstrated that in up to $14 \%$ of patients with a Glasgow Coma Scale of 14, CT scans showed intracranial lesions. ${ }^{15,16}$ Additional CT angiography should be performed in penetrating injuries with hematomas or suspected vascular lesions. ${ }^{8}$

Performing additional diagnostics may lead to therapeutic intervention. Without surgical removal of the foreign body, our patient would have been at increased risk for morbidity and mortality ${ }^{7}$ due to bleeding from the sagittal sinus, increasing intracranial pressure, or infections of the open wound. Nevertheless, surgical removal of the foreign body may itself provoke secondary parenchymal and vascular injuries. ${ }^{7,17}$ For instance, a neuroimaging-based approach allows for the reconstruction of the local anatomical structures, ${ }^{5-7,18}$ thus minimizing the risk of surgically induced secondary brain injuries.

\section{Conclusion}

Many guidelines exist on how to handle different kinds of traumatic brain injuries. ${ }^{8-10,13,14,16,19}$ Considering the great variability of trauma mechanisms and possible associated neurological deficits, each patient should be treated individually.

If the cause of the trauma is not obvious, the patient should be admitted into a neurosurgical unit for adequate surgical treatment. ${ }^{9}$ Unclear neurological status should indicate that neuroimaging (eg, CT) is necessary, especially in children, ${ }^{19}$ and that additional imaging (eg, CT angiography, MRI) should be performed if needed. Reducing the risk of a neurological deficit is of the highest priority for the patient, and thus a diagnostic CT scan may be warranted.

\section{Disclosure}

The authors report no conflicts of interest or breach of ethical adherence in this work. The authors did not receive any funding for this work from the National Institutes of Health, the Welcome Trust, the Howard Hughes Medical Institute, or any additional foundations.

\section{References}

1. Turbin RE, Maxwell DN, Langer PD, et al. Patterns of transorbital intracranial injury: a review and comparison of occult and non-occult cases. Surv Ophthalmol. 2006;51(5):449-460.

2. Youssef AS, Morgan JM, Padhya T, Vale FL. Penetrating craniofacial injury inflicted by a knife. J Trauma. 2008;64(6):1622-1624.

3. Ardill W, Gidado S. Penetrating head wound: a remarkable case. Surg Neurol. 2003;60(2):120-123.

4. Bhootra BL. Retained intra cranial blade - medicolegal perspectives. J Forensic Leg Med. 2007;14(1):31-34.

5. Herring CJ, Lumsden AB, Tindall SC. Transcranial stab wounds: a report of three cases and suggestions for management. Neurosurgery. 1988;23(5):658-662

6. Iwakura M, Kawaguchi T, Hosoda K, et al. Knife blade penetrating stab wound to the brain - case report. Neurol Med Chir (Tokyo) 2005;45(3):172-175. 
7. Taylor AG, Peter JC. Patients with retained transcranial knife blades: a high-risk group. J Neurosurg. 1997;87(4):512-515.

8. Maas AI, Stocchetti N, Bullock R. Moderate and severe traumatic brain injury in adults. Lancet Neurol. 2008;7(8):728-741.

9. Mendelow AD, Timothy J, Steers JW, et al. Management of patients with head injury. Lancet. 2008;372(9639):685-687.

10. Saatman KE, Duhaime AC, Bullock R, Maas AI, Valadka A, Manley GT; Workshop Scientific Team and Advisory Panel Members. Classification of traumatic brain injury for targeted therapies. JNeurotrauma. 2008;2(7): 719-738.

11. Bauer M, Patzelt D. Intracranial stab injuries: case report and case study. Forensic Sci Int. 2002;129(2):122-127.

12. Davis NL, Kahana T, Hiss J. Souvenir knife: a retained transcranial knife blade. Am J Forensic Med Pathol. 2004;25(3):259-261.

13. Ruff RM, Iverson GL, Barth JT, Bush SS, Broshek DK; NAN Policy and Planning Committee. Recommendations for diagnosing a mild traumatic brain injury: a National Academy of Neuropsychology education paper. Arch Clin Neuropsychol. 2009;24(1):3-10.
14. Teasdale G, Jennett B. Assessment of coma and impaired consciousness. A practical scale. Lancet. 1974;2(7872):81-84.

15. Lesieur O, Verrier V, Lequeux B, Lempereur M, Picquenot E. Retained knife blade: an unusual cause for headache following massive alcohol intake. Emerg Med J. 2006;23(2):e13.

16. Smits M, Dippel DW, Steyerberg EW, et al. Predicting intracranial traumatic findings on computed tomography in patients with minor head injury: the CHIP prediction rule. Ann Intern Med. 2007;146(6):397-405.

17. Pascual-Castroviejo I, Pascual-Pascual SI, Viano J. Diplegia due to transcranial knife-blade injury in a 20-month-old child. J Child Neurol. 2006;21(4):340-341.

18. Haworth CS, de Villiers JC. Stab wounds to the temporal fossa. Neurosurgery. 1988;23(4):431-435.

19. Willis AP, Latif SA, Chandratre S, Stanhope B, Johnson K. Not a NICE CT protocol for the acutely head injured child. Clin Radiol. 2008;63(2):165-169.
International Journal of General Medicine

\section{Publish your work in this journal}

The International Journal of General Medicine is an international, peer-reviewed open-access journal that focuses on general and internal medicine, pathogenesis, epidemiology, diagnosis, monitoring and treatment protocols. The journal is characterized by the rapid reporting of reviews, original research and clinical studies across all disease areas.

\section{Dovepress}

A key focus is the elucidation of disease processes and management protocols resulting in improved outcomes for the patient.The manuscript management system is completely online and includes a very quick and fair peer-review system. Visit http://www.dovepress.com/ testimonials.php to read real quotes from published authors. 